\title{
Intense Microsecond Electron Pulses from a Schottky Emitter
}

Gabriele Bongiovanni, Pavel K. Olshin, Marcel Drabbels, and Ulrich J. Lorenz

Affiliation: Laboratory of Molecular Nanodynamics, École Polytechnique Fédérale de Lausanne, 1015

Lausanne, Switzerland

* To whom correspondence should be addressed. E-mail: ulrich.lorenz@epfl.ch 


\begin{abstract}
Thanks to their high brightness, field emitters are the electron sources of choice in most high-end electron microscopes. Under typical operating conditions, the available emission current from these emitters is largely limited by practical considerations, and extracting significantly larger currents is usually not possible without reducing the lifetime of the emitter or even damaging it. Such limitations may however not apply if the emitter is only briefly subjected to extreme operating conditions, so that damage can be outrun. Here, we demonstrate that it is possible to temporarily operate a Schottky emitter far outside its stable operating regime and significantly increase its emission current. We do so by locally heating the tip of the emitter with a microsecond laser pulse, which boosts the emission current by a factor of 3.7 , to nearly $450 \mu \mathrm{A}$. We believe that the generation of intense, microsecond electron pulses from a field emitter will particularly benefit atomic-resolution imaging of fast processes that occur on the microsecond timescale.
\end{abstract}


Field emitters have greatly advanced a range of applications in electron microscopy that particularly benefit from their high brightness and coherence. ${ }^{1-6}$ More recently, they have also found application in the rapidly developing field of time-resolved electron microscopy, ${ }^{7-12}$ enabling for example the coherent manipulation of single-electron wavepackets with laser fields ${ }^{13}$ or time-resolved holography..$^{9,12}$ While field emitters made from carbon nanotubes, ${ }^{14} \mathrm{LaB}_{6}$ nanowires, ${ }^{15}$ or single atoms ${ }^{16}$ have set brightness records, tungsten field emitters are most widely used, as they offer stable operation over extended periods of time, as well as a lifetime of years. ${ }^{17,18}$ Schottky emitters consist of a single-crystalline tungsten to which a high extraction field is applied. They are coated with a layer of zirconium oxide to reduce the work function of the (100) emitting surface, ${ }^{3}$ and are typically operated at temperatures up to $1800 \mathrm{~K}$. This also reduces the buildup of contaminations and allows the zirconia layer on the emitting surface to continuously be replenished through diffusion from a reservoir on the body of the emitter. ${ }^{19}$

For many experiments with high time-resolution, in particular for imaging with short electron pulses, the available probe current is a critical figure of merit that limits the amount of information that can be obtained in a short amount of time. ${ }^{20,21}$ Given the excellent source properties of Schottky emitters, it is therefore tempting to try and extract larger currents. However, practical considerations limit the ability to do so by simply raising the extraction voltage or the operating temperature. At high extraction fields, tungsten atoms migrate towards the tip, which leads to unstable emission and causes the end facet to grow, ultimately shortening the lifetime of the emitter. $22,3,17,18$ The tip geometry is only stable over extended periods of time if the pull of the extraction field is exactly counterbalanced by the surface tension of the tip, which drives atoms to migrate in the opposite direction. ${ }^{22,3,17,18}$ For a typical gun geometry, the stable extraction voltage is about $3-4 \mathrm{kV} .{ }^{22}$ It is also impractical to raise the temperature of the emitter significantly above $1800 \mathrm{~K}$, where evaporation rapidly depletes the zirconium oxide reservoir and thus shortens the lifetime of the emitter. ${ }^{3}$ Above $1950 \mathrm{~K}$, the emission current begins to drop, as the zirconia coverage of the emitting surface decreases, ${ }^{22}$ and at $2400 \mathrm{~K}$, the zirconium oxide layer evaporates entirely, making operation impossible. ${ }^{23}$ It has been reported that under extreme conditions, at $1950 \mathrm{~K}$ and with about twice the stable extraction voltage, a stable emission current of $500 \mu \mathrm{A}$ can be sustained for several days. ${ }^{22,24}$ However, under practical operating conditions, the emission current is typically about $100 \mu \mathrm{A} .^{22}$ 
Here, we demonstrate that it is possible to boost emission from a Schottky emitter by temporarily operating it at extreme temperatures. When we heat the emitter tip with a microsecond laser pulse to an estimated temperature of over $2500 \mathrm{~K}$, the emission current increases to nearly $450 \mu \mathrm{A}$. We envision that in combination with a beam blanker, this will enable the generation of intense microsecond electron pulses of high brightness. While femtosecond and nanosecond photoelectron pulses from field emitters have previously been demonstrated, ${ }^{8,9,25-28}$ photoemission does not provide a convenient mechanism to access the microsecond timescale. Because of the low photoelectron yields involved, the laser powers required to sustain a large photoemission current would rapidly heat the emitter to temperatures at which thermal emission vastly exceeds photoemission. ${ }^{29}$ Our experiments demonstrate the generation of intense microsecond electron pulses from a field emitter, which will particularly benefit atomic-resolution imaging of fast processes occurring on the microsecond timescale.

Experiments were carried out with a modified JEOL Schottky field emission gun, whose emitter assembly is illustrated in Fig. 1a. The emitter (430 nm tip radius) protrudes from a hole in the suppressor, which is held at $-300 \mathrm{~V}$ with respect to the emitter. The extractor is located at a distance of $350 \mu \mathrm{m}$ from the tip and biased to $+3.0 \mathrm{kV}$. The assembly is completed by the so-called anode 2 , which serves as an electrostatic lens (typically $+7.8 \mathrm{kV}$ ). Unless otherwise noted, the emitter temperature is set to $1720 \mathrm{~K}$, the operating temperature recommended by the manufacturer of the gun, at which we obtain an emission current of $120 \mu \mathrm{A}$. In order to transiently boost emission, we heat the emitter tip with a high-power continuous laser (532 nm wavelength), as illustrated in Fig. 1b. The laser beam is chopped into microsecond pulses with an acousto-optic modulator, expanded, and focused onto the tip of the emitter (17 $\mu \mathrm{m}$ FWHM laser spot size), which it strikes under an angle of $16^{\circ}$ with respect to the electron optical axis. A piezo-controlled mirror facilitates alignment. The reported laser powers refer to the power that reaches the emitter, where we have accounted for losses on optics. For the experiments reported in Fig. 2, the electron gun was mounted on a JEOL 2010F transmission electron microscope and operated at $200 \mathrm{kV}$ accelerating voltage. The probe current was measured on the phosphor screen of the microscope, with a fast transimpedance amplifier connected to record the temporal structure of the electron pulses. For the experiments in Fig. 4, the emitter assembly was removed from the gun and mounted in a dedicated vacuum chamber. The electron beam was monitored on a phosphor screen at a distance of $25 \mathrm{~cm}$ from the emitter. 
Figure $2 \mathrm{a}$ demonstrates that laser heating the tip of the emitter can be used to significantly boost electron emission for a duration of hundreds of microseconds. Under irradiation with a $100 \mu \mathrm{s}$ laser pulse (red curve, $1 \mathrm{kHz}$ repetition rate), the probe current (blue) increases rapidly, by more than three times at the end of the laser pulse, before it slowly returns to its initial value. We obtain a rise time of the electron pulse of $11 \mu$ s from a fit with the sum of an exponential and a linear function, which appears to give a good description for a range of pulse lengths. The tail of the pulse follows a biexponential decay with timescales of $14 \mu$ s and $85 \mu$ s (75\% and $25 \%$ weight). For simplicity, we report $80 \%$ to $20 \%$ fall times in the following, for which we measure $36 \mu$ s here.

Figure $2 \mathrm{~b}$ shows that the peak probe current increases linearly with laser power (100 $\mu$ s pulses, $20 \mathrm{~Hz})$, reaching $210 \mathrm{nA}$ at $0.64 \mathrm{~W}$, which corresponds to an enhancement of 3.7 times and an emission current of nearly $450 \mu \mathrm{A}$. The linear increase of the probe current is consistent with the high-temperature behavior of Schottky emitters. While below $1600 \mathrm{~K}$, the emission current follows Schottky theory and grows exponentially with temperature, ${ }^{1}$ between $1600 \mathrm{~K}$ and $1850 \mathrm{~K}$, it only increases linearly, ${ }^{3}$ which is due to a linear increase of the work function in this temperature range. ${ }^{23}$ For our emitter, the probe current increases by $20 \mathrm{nA}$ per $100 \mathrm{~K}$, which allows us to estimate the peak temperature of the tip. (Fig. 2b, top axis). For the highest laser power, the tip reaches over $2500 \mathrm{~K}$, vastly exceeding the recommended maximum operating temperature $(1850 \mathrm{~K})$, and well above the temperature at which zirconium oxide is completely removed from a tungsten surface $(2400 \mathrm{~K}) .{ }^{23}$ While continuous operation at such an extreme temperature would be all but impossible, we are evidently able to outrun the evaporation of zirconia from the end facet. This process likely requires tens of seconds, the time it takes the emission current to stabilize after a sudden temperature change. ${ }^{3}$ We note that for high current densities, Coulomb interactions ultimately limit the brightness ${ }^{24,30}$. We therefore expect the increase in brightness to be lower than the increase in current.

If the emitter is continuously exposed to intense laser pulses, the peak probe current slowly decreases over time, as shown in Fig. 2c. Under irradiation with $100 \mu$ s pulses of $0.44 \mathrm{~W}$ at $1 \mathrm{kHz}$ repetition rate, the enhancement of the peak probe current is initially three, but then slowly drops to two, with a time constant of one hour. Apparently, under these conditions, the rate at which the laser pulses remove 
zirconium oxide from the end facet exceeds the rate at which it can be replenished through diffusion. The zirconium oxide coverage therefore decreases, and with it the peak current, until an equilibrium is established in which both rates are equal. We note that after we stop irradiating it, the emitter slowly recovers, and the peak probe current returns to its initial value. Moreover, we do not observe any significant changes in the emission characteristics over time, suggesting that the tip shape remains stable, as is expected when the emitter is operated with a stable extraction voltage. At higher repetition rates, the enhancement of the peak probe current is initially the same, with the drop occurring faster accordingly.

Finite element simulations (Comsol Multiphysics) provide additional insights into the heating and cooling dynamics of the emitter. As illustrated in Fig. 3a, we model an emitter of typical dimensions with the thermal properties of tungsten ${ }^{31}$ and an initial temperature of $1720 \mathrm{~K}$. A tip of $500 \mathrm{~nm}$ radius tops a $1.4 \mu \mathrm{m}$ long cylindrical shaft, which widens in a $300 \mu \mathrm{m}$ long conical section to the diameter of the body of the emitter $(125 \mu \mathrm{m})$. The emitter is heated by a Gaussian laser beam (17 $\mu \mathrm{m}$ FWHM) that is centered onto the base of the shaft and strikes it under an angle of $16^{\circ}$ with respect to the electron optical axis (inset). We neglect the variation of the absorbance with local incidence angle and instead assume an average absorbance of $21 \%{ }^{32}$

Figure $3 \mathrm{~b}$ shows the temperature evolution of the emitting surface under irradiation with a $0.8 \mathrm{~W}$ laser pulse of $100 \mu$ s duration. We obtain heating and cooling times of $7 \mu$ s and $33 \mu \mathrm{s}$, respectively, in reasonable agreement with the experiment. The temperature jump is somewhat lower, most likely reflecting differences between the simulated and actual emitter geometry. The surface temperature distribution at the end of the laser pulse (Fig. 3a, color) reveals that only the very tip of the emitter is being heated up significantly, while the temperature of the emitter body remains unchanged. At a distance of only $30 \mu \mathrm{m}$ from the tip, the laser induced temperature jump decreases by half. Such very localized heating is favorable for the purpose of creating intense electron pulses, since loss of zirconium oxide that inevitably occurs at extreme temperatures is mostly limited to the tip. Diffusion from adjacent areas can therefore rapidly replenish the zirconium oxide layer on the end facet. In contrast, heating the entire emitter to extreme temperatures would remove zirconium oxide from its whole surface, thus making operation impossible. 
The electron yield and pulse shape are sensitive to the alignment of the laser. This is evident in Fig. 4a, which displays the number of electrons emitted per pulse as the laser beam is being rastered over the emitter by means of the piezo-controlled mirror (see Fig. $1 b ; 100 \mu$ s laser pulses at $0.2 \mathrm{~W}$, with the emitter temperature lowered to $1400 \mathrm{~K}$, so that no emission occurs without laser). For reference, the orientation of the emitter is indicated above the plot, together with the directions in which the laser beam is scanned. The electron yield roughly maps out the profile of the emitter, with the conical body faintly visible on the left, and the tip marked by an intense maximum in the center. This maximum appears elongated, with a FWHM of $218 \mu \mathrm{m}$ along the emitter axis, and $17 \mu \mathrm{m}$ orthogonal to it. Since the tip of the emitter is small ( $430 \mathrm{~nm}$ radius), we infer that the laser beam diameter at the emitter is about $17 \mu \mathrm{m}$ FWHM. As the laser is scanned towards the emitter body, it has to heat up a larger volume, which results in a lower temperature of the end facet and thus a lower emission current. For the same reason, we also observe slower rise and fall times with the laser aligned to the conical part of the emitter. We conclude that a fast response of the emitter as well as large peak currents are obtained with the laser focus centered near the tip. Under such conditions, the laser spot size can be easily minimized by monitoring the emission current. When we change the length of the beam expanding telescope (see Fig. 1b), the peak current goes through a maximum (Fig. 4b), which indicates optimum focusing conditions. Focusing the laser as tightly as possible is desirable in order to obtain the most localized heating and thus the fastest thermal response of the emitter.

In the future, we envision adding a beam blanker ${ }^{21}$ to our microscope in order to chop the laser boosted electron beam into pulses of desired duration. The generation of intense microsecond electron pulses from a field emitter promises to enable atomic-resolution single-shot ${ }^{33,34}$ as well as stroboscopic imaging of fast processes occurring on the microsecond timescale. A particular appeal of such an approach lies in the fact that it would allow one to align and operate the microscope under standard conditions and then switch on the fly to pulsed operation with a laser boosted electron beam. The laser power should be chosen based on the desired application. For single-shot imaging or stroboscopic imaging with a limited number of electron pulses, it is desirable to extract the largest number of electrons and therefore use the highest laser power. Pulsed operation over extended periods of time may require reducing the laser power in order to limit the removal or zirconia from the end facet. Finally, we note that emission 
currents can also be further increased by raising the extraction voltage, which, however, will reduce the lifetime of the emitter.

\section{Acknowledgments}

This work was supported by the ERC Starting Grant 759145 and by Swiss National Science Foundation Grants PP00P2_163681 and 206021_183295.

\section{Data availability statement}

The data that support the findings of this study are available from the corresponding author upon reasonable request. 


\begin{abstract}
References
${ }^{1}$ L. Swanson and G. Schwind, in Handbook of Charged Particles Optics, 2nd ed. (CRC Press, 2008), pp. 1-28.

${ }^{2}$ L.W. Swanson and G.A. Schwind, Adv. Imaging Electron Phys. 159, 63 (2009).

${ }^{3}$ M.S. Bronsgeest, Physics of Schottky Electron Sources, (Jenny Stanford Publishing 2014).

${ }^{4}$ M.T. Otten and W.M.J. Coene, Ultramicroscopy 48, 77 (1993).

${ }^{5}$ A. Bogner, P.-H. Jouneau, G. Thollet, D. Basset, and C. Gauthier, Micron 38, 390 (2007).

${ }^{6}$ H. Lichte and M. Lehmann, Rep. Prog. Phys. 71, (2008).

${ }^{7}$ O.F. Mohammed, D.-S. Yang, S.K. Pal, and A.H. Zewail, J Am Chem Soc 133, 7708 (2011).

${ }^{8}$ A. Feist, N. Bach, N. Rubiano da Silva, T. Danz, M. Möller, K.E. Priebe, T. Domröse, J.G. Gatzmann,

S. Rost, J. Schauss, S. Strauch, R. Bormann, M. Sivis, S. Schäfer, and C. Ropers, Ultramicroscopy
\end{abstract} 176, 63 (2017).

${ }^{9}$ F. Houdellier, G.M. Caruso, S. Weber, M. Kociak, and A. Arbouet, Ultramicroscopy 186, 128 (2018).

${ }^{10}$ P. Das, J.D. Blazit, M. Tencé, L.F. Zagonel, Y. Auad, Y.H. Lee, X.Y. Ling, A. Losquin, C. Colliex, O. Stéphan, F.J. García de Abajo, and M. Kociak, Ultramicroscopy 203, 44 (2019).

${ }^{11}$ C. Zhu, D. Zheng, H. Wang, M. Zhang, Z. Li, S. Sun, P. Xu, H. Tian, Z. Li, H. Yang, and J. Li, Ultramicroscopy 209, 112887 (2020).

12 T. Niermann, M. Lehmann, and T. Wagner, Ultramicroscopy 182, 54 (2017).

${ }^{13}$ A. Feist, K.E. Echternkamp, J. Schauss, S.V. Yalunin, S. Schafer, and C. Ropers, Nature 521, 200 (2015).

${ }^{14}$ F. Houdellier, L. de Knoop, C. Gatel, A. Masseboeuf, S. Mamishin, Y. Taniguchi, M. Delmas, M. Monthioux, M.J. Hytch, and E. Snoeck, Ultramicroscopy 151, 107 (2015).

${ }^{15}$ H. Zhang, J. Tang, J. Yuan, Y. Yamauchi, T.T. Suzuki, N. Shinya, K. Nakajima, and L.-C. Qin, Nat. Nanotechnol. 11, 273 (2016).

${ }^{16}$ T. Ishikawa, T. Urata, B. Cho, E. Rokuta, C. Oshima, Y. Terui, H. Saito, A. Yonezawa, and T.T. Tsong, Appl. Phys. Lett. 90, 143120 (2007).

17 K. Liu, G.A. Schwind, L.W. Swanson, and J.A. Campbell, J. Vac. Sci. Technol. B Nanotechnol. Microelectron. Mater. Process. Meas. Phenom. 28, C6C26 (2010).

${ }^{18}$ A. Bahm, G. Schwind, and L. Swanson, J. Appl. Phys. 110, 054322 (2011).

${ }^{19}$ P.C. Tiemeijer, M. Bischoff, B. Freitag, and C. Kisielowski, Ultramicroscopy 114, 72 (2012). 
${ }^{20}$ M.R. Armstrong, K. Boyden, N.D. Browning, G.H. Campbell, J.D. Colvin, W.J. DeHope, A.M. Frank, D.J. Gibson, F. Hartemann, J.S. Kim, W.E. King, T.B. LaGrange, B.J. Pyke, B.W. Reed, R.M. Shuttlesworth, B.C. Stuart, and B.R. Torralva, Ultramicroscopy 107, 356 (2007).

${ }^{21}$ L. Zhang, J.P. Hoogenboom, B. Cook, and P. Kruit, Struct. Dyn. 6, 051501 (2019).

${ }^{22}$ A.H.V. van Veen, C.W. Hagen, J.E. Barth, and P. Kruit, J. Vac. Sci. Technol. B Microelectron. Nanometer Struct. Process. Meas. Phenom. 19, 2038 (2001).

${ }^{23}$ L.R. Danielson, J. Appl. Phys. 52, 6769 (1981).

${ }^{24}$ B. Cook, T. Verduin, C.W. Hagen, and P. Kruit, J. Vac. Sci. Technol. B 28, C6c74 (2010).

${ }^{25}$ P. Hommelhoff, C. Kealhofer, and M.A. Kasevich, Phys. Rev. Lett. 97, (2006).

${ }^{26}$ D. Ehberger, J. Hammer, M. Eisele, M. Kruger, J. Noe, A. Hogele, and P. Hommelhoff, Phys. Rev. Lett. 114, (2015).

27 J. Hoffrogge, J.P. Stein, M. Kruger, M. Forster, J. Hammer, D. Ehberger, P. Baum, and P. Hommelhoff, J. Appl. Phys. 115, (2014).

${ }^{28}$ R. Bormann, S. Strauch, S. Schäfer, and C. Ropers, J. Appl. Phys. 118, 173105 (2015).

${ }^{29}$ B. Cook, M. Bronsgeest, K. Hagen, and P. Kruit, Ultramicroscopy 109, 403 (2009).

${ }^{30}$ N. Bach, T. Domröse, A. Feist, T. Rittmann, S. Strauch, C. Ropers, and S. Schäfer, Struct. Dyn. 6, 014301 (2019).

${ }^{31}$ P. Tolias, Nucl. Mater. Energy 13, 42 (2017).

${ }^{32}$ M.A. Ordal, R.J. Bell, R.W. Alexander, L.A. Newquist, and M.R. Querry, Appl. Opt. 27, 1203 (1988).

${ }^{33}$ N.D. Browning, M.A. Bonds, G.H. Campbell, J.E. Evans, T. LaGrange, K.L. Jungjohann, D.J. Masiel,

J. McKeown, S. Mehraeen, B.W. Reed, and M. Santala, Curr. Opin. Solid State Mater. Sci. 16, 23 (2012).

${ }^{34}$ M. Picher, K. Bücker, T. LaGrange, and F. Banhart, Ultramicroscopy 188, 41 (2018). 
Figure captions

Figure 1. Illustration of the experimental setup. (a) Geometry of the emitter assembly. Operating voltages are indicated relative to the emitter. (b) The emission current is boosted by heating the tip of the emitter with microsecond laser pulses.

Figure 2. Generation of intense electron pulses. (a) Temporal evolution of the electron probe current (blue) when the emitter tip is illuminated with $100 \mu$ s laser pulses (red). (b) Peak probe current as a function of laser power. (c) Measurement of the long-term stability of the peak probe current at high repetition rate $(0.39 \mathrm{~W}, 100 \mu$ s pulses, $1 \mathrm{kHz})$.

Figure 3. Simulation of the temperature evolution of the emitter under laser illumination. (a) Surface temperature of the emitter after illumination with a $100 \mu$ s laser pulse. The laser beam (17 $\mu \mathrm{m}$ FWHM) is indicated with a green arrow. (b) Temperature evolution of the emitting surface (blue) during illumination with a $100 \mu$ s laser pulse (red).

Figure 4. Electron yield as a function of the laser focus position and beam diameter. (a) Number of electrons as a function of the laser focus position. The orientation of the emitter is illustrated above the plot. (b) Peak probe current as a function of the length of the beam expanding telescope (see Fig. 1b). 

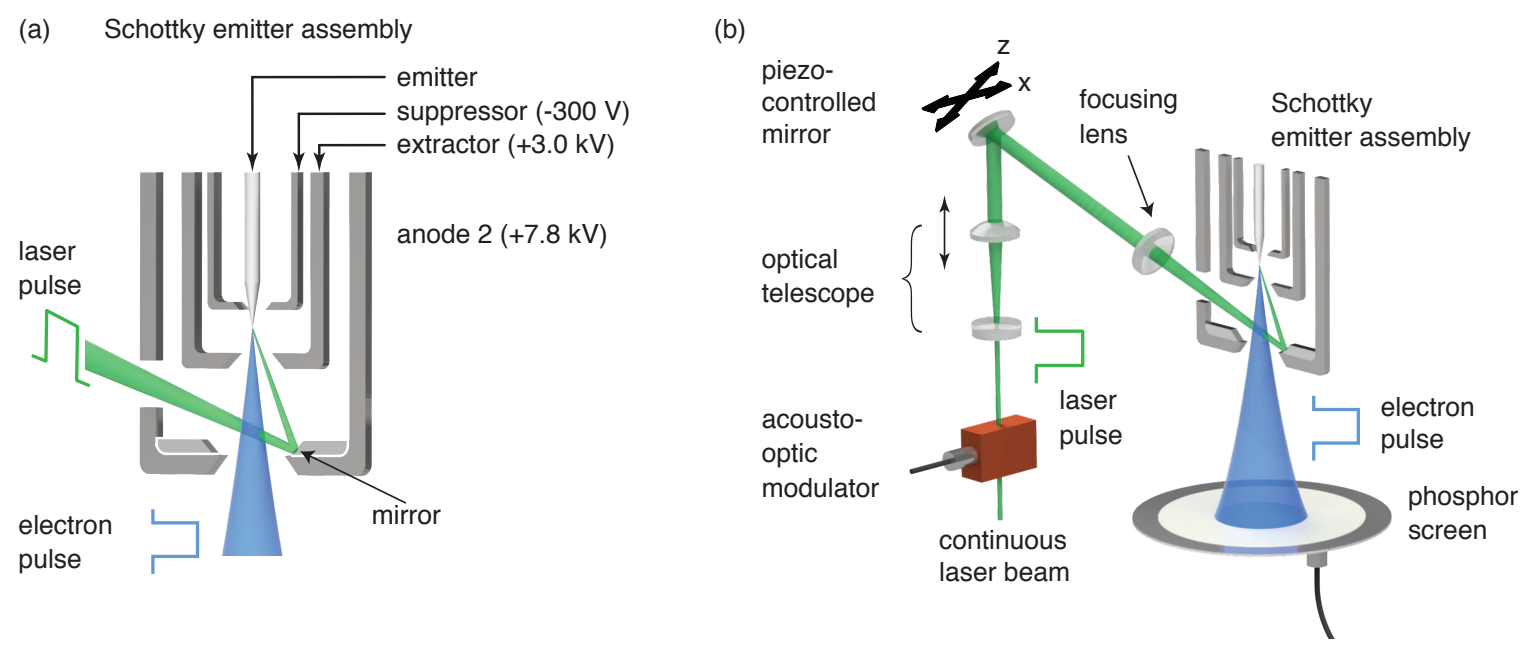

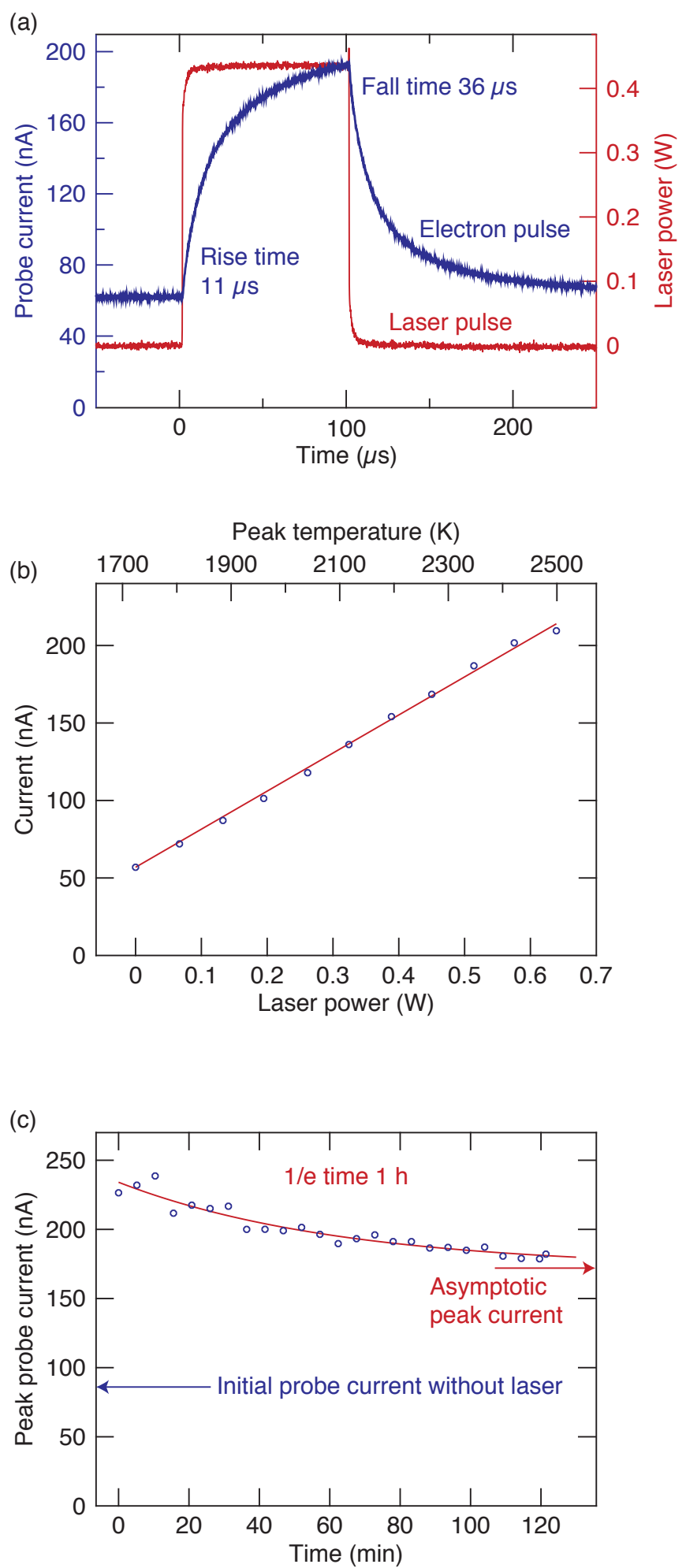
(a)

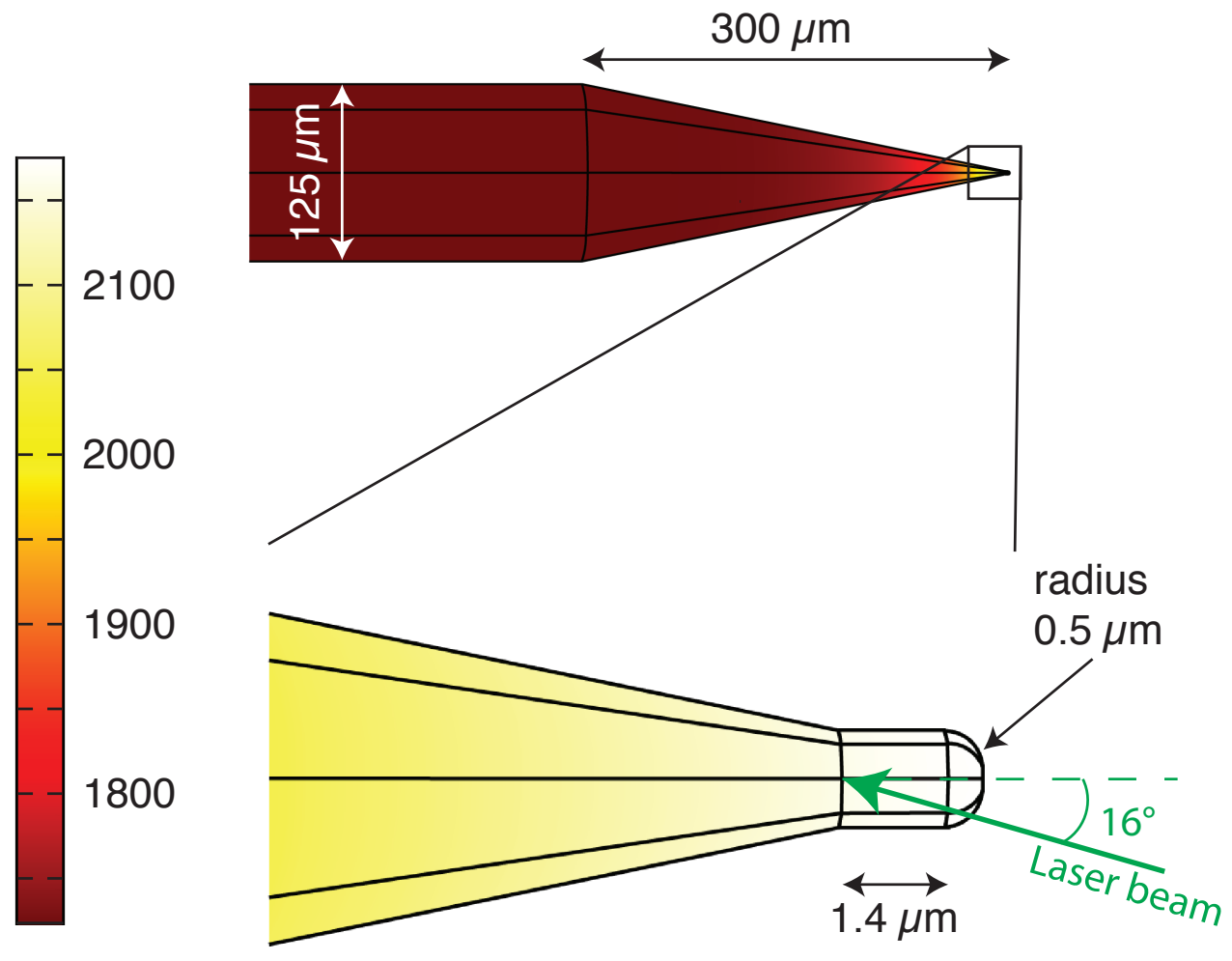

(b)

Surface temperature $(\mathrm{K})$

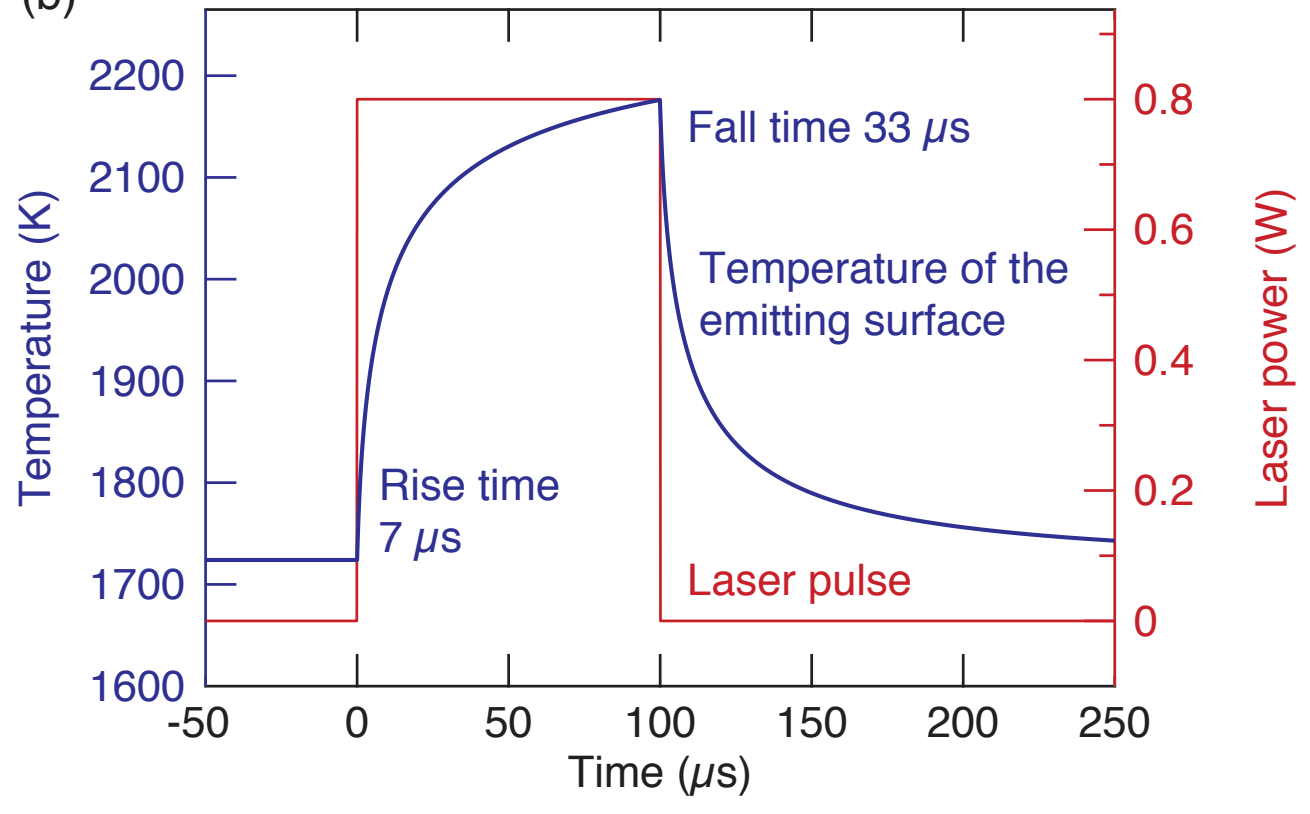


(a)

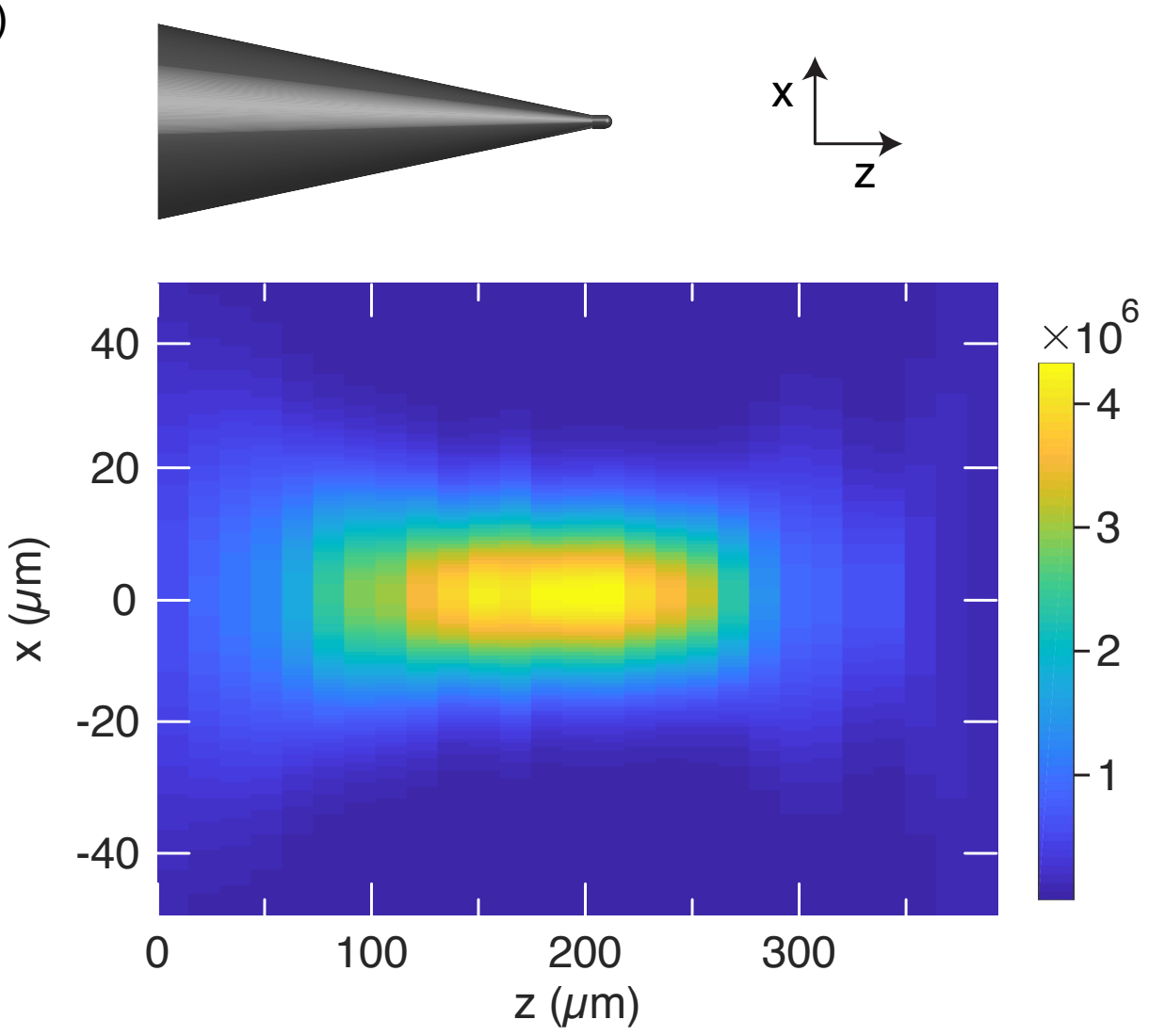

(b)

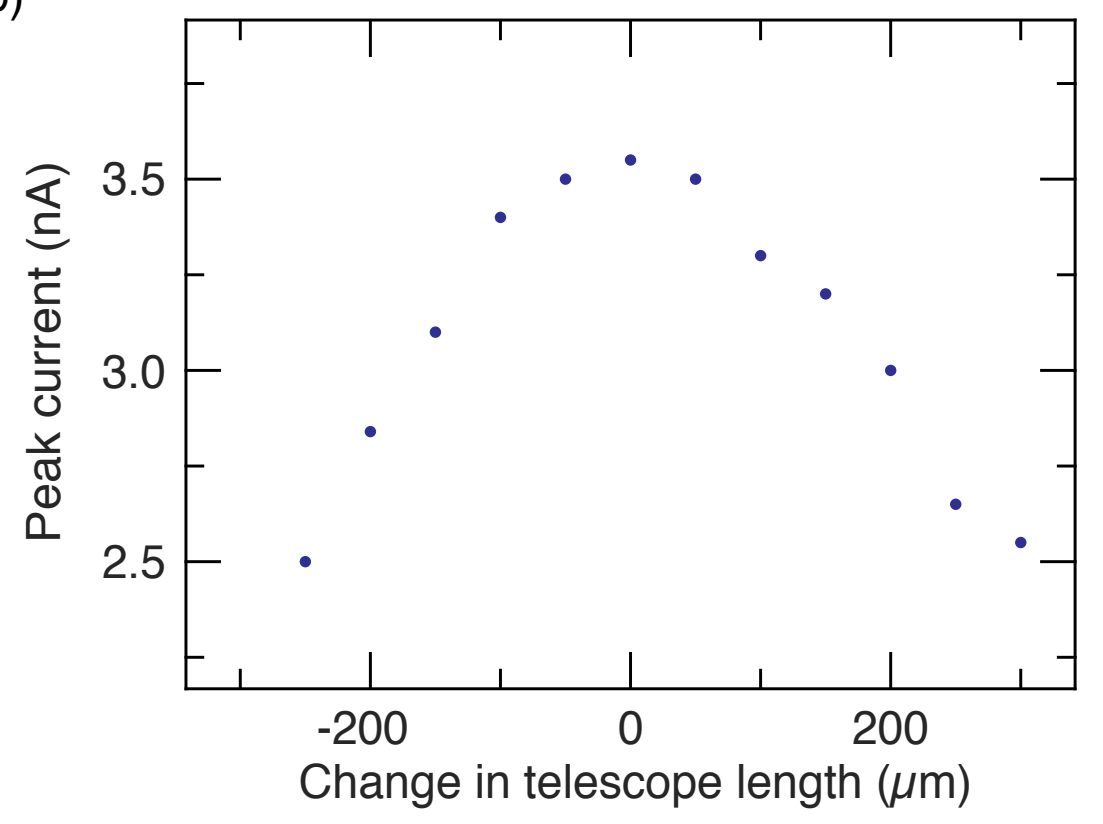

